\title{
Reversibility in radionuclide/bentonite bulk and colloidal ternary systems
}

\author{
Nick Sherriff ${ }^{1}$, Ragiab Issa ${ }^{1}$, Katherine Morris ${ }^{2}$, Francis Livens ${ }^{1,2}$, Sarah Heath $^{1}$ and Nick Bryan ${ }^{3, *}$ \\ 1 Centre for Radiochemistry Research, School of Chemistry, University of Manchester, Manchester M13 9PL, UK \\ 2 Research Centre for Radwaste and Decommissioning, School of Earth, Atmospheric and Environmental Sciences, \\ University of Manchester, Manchester M13 9PL, UK \\ 3 National Nuclear Laboratory, 5th Floor, Chadwick House, Birchwood, Warrington WA3 6AE, UK
}

[Received 10 October 2014; Accepted 16 June 2015; Associate Editor: Nicholas Evans]

\section{ABSTRACT}

Ternary systems of ${ }^{152} \mathrm{Eu}(\mathrm{III})$, bulk bentonite and ethylenediaminetetraacetic acid (EDTA) $([\mathrm{Eu}]=7.9 \times$ $10^{-10} \mathrm{M} ; \mathrm{pH}=6.0-7.0$ ) have been studied. Without EDTA, there was slow uptake in a two-stage process, with initial rapid sorption of $\mathrm{Eu}(\mathrm{III})(96 \%)$, followed by slower uptake of a much smaller fraction $(3.0 \%$ over a period of one month). The reversibility of Eu(III) binding was tested by allowing $\mathrm{Eu}(\mathrm{III})$ to sorb to bentonite for 1-322 days. EDTA was added to the pre-equilibrated Eu bentonite systems at $0.01 \mathrm{M}$, a concentration that was sufficient to suppress sorption in a system where EDTA was present prior to the contact of $\mathrm{Eu}$ (III) with bentonite. A fraction of the Eu was released instantaneously (30-50\%), but a significant amount remained bound. With time, the amount of $\mathrm{Eu}(\mathrm{III})$ retained by the bentonite reduced, with a slow fraction dissociation rate constant of approximately $4.3 \times 10^{-8} \mathrm{~s}^{-1}$ (values in the range $2.2 \times$ $10^{-8}-1.0 \times 10^{-7} \mathrm{~s}^{-1}$ ) for pre-equilibration times $\geq 7$ days. Eventually, the amount of Eu(III) remaining bound to the bentonite was within error of that when EDTA was present prior to contact $(4.5 \% \pm 0.6)$, although in systems with pre-equilibration times $>100$ days, full release took up to 500 days. Europium interactions with colloidal bentonite were also studied, and the dissociation rate constant measured by a resin competition method. For the colloids, more Eu was found in the slowly dissociating fraction $(60-70 \%)$, but the first-order dissociation rate constant was faster, with an average rate constant of $8.8 \times 10^{-7} \mathrm{~s}^{-1}$ and a range of $7.7 \times 10^{-7}-9.5 \times 10^{-7} \mathrm{~s}^{-1}$. For both bulk and colloidal bentonite, although slow dissociation was observed for $\mathrm{Eu}(\mathrm{III})$, there was no convincing evidence for 'irreversible' binding.

KEYwords: ternary systems, bentonite, ethylenediaminetetraacetic acid, EDTA, europium, ${ }^{152} \mathrm{Eu}(\mathrm{III})$, reversibility, dissociation rate constant.

\section{Introduction}

STUDIES suggest that for colloidal transport to be significant, colloids must bind radionuclides irreversibily, as any reversibly bound radionuclides

* E-mail: nick.bryan@nnl.co.uk

DOI: 10.1180/minmag.2015.079.06

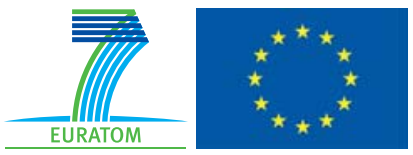

would be removed quickly by the available rock surface binding sites, whereas 'irreversibly' bound radionuclides would be transported with the colloids (Mori et al., 2003). In fact, the situation is more complex than this, because there is no simple division between reversibly and irreversibly bound metal ions. Calculations have shown that the slow dissociation of radionuclides from colloids (i.e. short of 'irreversibility') can facilitate transport.

The publication of this research has been funded by the European Union's European Atomic Energy Community's (Euratom) Seventh Framework programme FP7 (2007-2013) under grant agreements $n^{\circ} 249396$, SecIGD, and n`323260, SecIGD2. 
The transport residence time only needs to be small compared to the half time for dissociation, with migration increasing with decreasing rate constant (Bryan et al., 2007).

The interactions of radionuclides with bulk and colloidal clays are important, because they have been suggested as a potential backfill material for a radioactive waste repository (Mori et al., 2003), and so the sorption of radionuclides by bulk bentonite, its constituents and other bulk clays have been studied extensively (Wold, 2010). However, only the reversibility of $\mathrm{Cs}(\mathrm{I})$ binding to bulk clays has been studied in detail (e.g. Comans, 1987; Comans et al., 1991; de Koning and Comans, 2004), although Ivanov et al. (2012) have reported no evidence for slow dissociation of uranyl from bulk bentonite.

Radionuclide sorption to bentonite colloids has also received attention; however, the dissociation kinetics have been studied barely at all (Wold, 2010). In conditions relevant to the vicinity of a clay buffered radioactive waste repository, Missana et al. (2008) found $>75 \%$ colloid bound for $\mathrm{Eu}(\mathrm{III})$ and $\mathrm{Pu}(\mathrm{IV})$, whilst Schäfer et al. (2004) found that approximately $80 \%$ of both Th(IV) and $\mathrm{Eu}(\mathrm{III})$ were colloid-associated. Huber et al. (2011) have reported competition experiments between bentonite colloids and fracture-filling material. Slow dissociation from the colloids was observed, and there was some evidence that the system took 7500 hours (313 days) to reach equilibrium: for Am(III), the rate constants were in the range $1-2.5 \times$ $10^{-6} \mathrm{~s}^{-1}$, whilst for $\mathrm{Pu}(\mathrm{IV})$, the range was $3.9 \times$ $10^{-7}-2.4 \times 10^{-6} \mathrm{~s}^{-1}$. Wold (2010) estimated representative first-order dissociation rate constants from sorption rate constants and $K_{\mathrm{d}}$ values for: $\mathrm{Pu}$ (IV) $1.2 \times 10^{-6} \mathrm{~s}^{-1} ; \quad A m(I I I) \quad 5.6 \times 10^{-7} \mathrm{~s}^{-1}$; $\mathrm{Np}(\mathrm{IV}) 1.2 \times 10^{-10} \mathrm{~s}^{-1} ; \mathrm{Cm}(\mathrm{III}) 1.7 \times 10^{-6} \mathrm{~s}^{-1}$; U(VI) $8.3 \times 10^{-7} \mathrm{~s}^{-1} ; \mathrm{Tc}(\mathrm{IV}) 1.75 \times 10^{-4}-4.2 \times$ $10^{-3} \mathrm{~s}^{-1}$. Geckeis et al. (2004) found that Am(III) and $\mathrm{Pu}(\mathrm{IV})$ transport through fractures could only be explained by slow dissociation from colloids, although the interactions were eventually reversible on a time scale of months.

Bouby et al. (2011) found that Cs(I) and U(VI) did not bind to bentonite colloids, but that the triand tetravalent f-block ions (Eu(III); Th(IV)) were strongly associated. For these two metal ions, they measured the time taken for complexation by humic acid to remove the metal from the bentonite. In the case of $\mathrm{Eu}(\mathrm{III})$, they found that although dissociation from the colloids was slow, equilibrium was eventually attained, and most of the $\mathrm{Eu}(\mathrm{III})$ dissociated from the bentonite and bound to the humic acid. However, in the case of Th(IV), even after three years, the system had not reached equilibrium.

In this study, we have determined the dissociation rate constants for $\mathrm{Eu}(\mathrm{III})$ from both bulk and colloidal bentonite. The study of the uptake of radionuclides by bentonite is relatively straightforward, and requires only that the radionuclide is added to the clay. However, to study the reversibility of the interaction, it is necessary to allow the radionuclide to interact with the bentonite for a known period of time before using a competing ligand or cation binding resin as a strong sink to 'pull' the radionuclide from the bentonite, so that the dissociation rate may be measured (e.g. Monsallier et al., 2003). Previous tri-valent f-element dissociation experiments have not considered dissociation from bulk bentonite, and those with colloidal bentonite have used ligands such as humic acid or complex natural solid competitors, such as fracture fill material, that could interact with the colloids. Other literature values are estimates based on sorption rates and distribution coefficients. The aim of this work is to measure directly the dissociation rate constants for bulk and colloidal bentonite using sinks that do not interact with bentonite: such data are useful in the prediction of radionuclide migration in environments where bentonite exists, such as deep geological disposal.

\section{Materials and methods}

The clay used in this work is a sodium Wyoming bentonite. All water was deionized (18 M $\Omega$ ) and all reagents were analytical grade. All experiments were repeated in triplicate.

\section{Bulk Eu dissociation experiments}

Bentonite clay $(0.5 \mathrm{~g})$ was added to tubes with deionized water $(8 \mathrm{ml}), \mathrm{NaClO}_{4}(1 \mathrm{ml}, 0.1 \mathrm{M})$ and ${ }^{152} \mathrm{Eu}(1 \mathrm{ml}, 1 \mathrm{kBq})$ to give a total Eu concentration of $7.9 \times 10^{-10} \mathrm{M}$; the system was then adjusted to pH $7( \pm 0.1)$. The clay was suspended (maximizing contact with the $\mathrm{Eu}(\mathrm{III})$ ). After a pre-equilibration period between 1 and 332 days, EDTA sodium salt was added to the system. Before the addition of EDTA, the tubes were centrifuged on a BOECO C-28A centrifuge (15 min, $4000 \mathrm{rpm}$, Sigma microcentrifuge) to remove particles larger than $2 \mu \mathrm{m}$. Once this was complete, the top $4 \mathrm{ml}$ of suspension in the tube was removed and distributed into $2 \mathrm{ml}$ containers. These were then centrifuged ( $35 \mathrm{~min}$, 
$14000 \mathrm{rpm}$ ); this removed all particles larger than $0.25 \mu \mathrm{m}$. Following centrifugation, $1 \mathrm{ml}$ of the solution was removed and replaced by EDTA solution, which when introduced to the $10 \mathrm{ml}$ system gave an EDTA concentration of $0.01 \mathrm{M}$. Once the EDTA was added, the tubes were rocked for one hour to re-suspend the clay. Following the re-suspension, and after a period of one day, the tubes were again centrifuged as before, and $1.5 \mathrm{ml}$ was removed for analysis by gamma ray spectrometry (Canberra 2020 coaxial HPGe gamma spectrometer with an Ortec 919E multi-channel analyser). The aliquots were then returned to the sample tube, the clay re-suspended and the sample stored for later analyses. In a separate experiment, the uptake of $\mathrm{Eu}$ on to bentonite was measured in the absence of EDTA, but under the same conditions. Also, an experiment was performed where EDTA was added to the Eu tracer solution, prior to any contact with the bentonite clay (this was to ensure that the EDTA could effectively compete with the clay for the $\mathrm{Eu}$ ).

\section{Colloid studies}

A stock solution of colloids (147 ppm) was prepared and colloid concentrations measured according to the method described by Bouby et al. (2011). An aliquot of the sample was diluted with deionized water ( $1 \mathrm{ml}$ colloid suspension, $9 \mathrm{ml}$ water), and $\mathrm{HNO}_{3}$ was added $(0.2 \mathrm{ml})$ prior to analysis. The $\mathrm{Al}$ and $\mathrm{Mg}$ concentrations were determined using ICPAES (inductively coupled plasma atomic emission spectroscopy) on a Perkin Elmer Optima 5300 dual view spectrometer.

\section{Size distributions}

Syringe filters and ultrafiltration membranes were used sequentially to filter the bentonite colloid stock (450, 200 and $100 \mathrm{~nm}$ PES filters). The filters and membranes were adjusted to the $\mathrm{pH}$ of the colloid suspension $(8.8 \pm 0.2)$ and pre-equilibrated for 68 hours prior to use. Ultrafiltration was performed under pressure (argon, 1.5 bar) through membranes (Millipore, polyethersulfone: 300,10 and $3 \mathrm{kDa}$ ). In a separate experiment, ${ }^{152} \mathrm{Eu}(3.5 \mathrm{ml}, 35 \mathrm{kBq})$ was added to a sample of the bentonite colloid stock $(3.5 \mathrm{ml})$ and left for 68 hours to equilibrate before filtration as above. The syringe filters and ultrafiltration membranes were pre-treated in $10^{-4} \mathrm{M}$ stable $\mathrm{Eu}\left(\mathrm{NO}_{3}\right)_{3}$ solution to prevent sorption of the radiotracer, according to the method of Pitois et al. (2008).

Dowex 50WX4-200 (H-form) resin was converted to its sodium form. $20 \mathrm{~g}$ of Dowex resin were added to a sintered column, and washed with: deionized water $(150 \mathrm{ml})$; $\mathrm{HCl}(2 \mathrm{M}, 500 \mathrm{ml})$; deionized water $(500 \mathrm{ml}) ; \mathrm{NaCl}(3 \mathrm{M}, 500 \mathrm{ml})$; $\mathrm{NaOH}(0.1 \mathrm{M}, 500 \mathrm{ml})$; and finally deionized water $(500 \mathrm{ml})$. Experiments showed that the colloid did not interact with the $\mathrm{pH}$ pre-conditioned resin (data not shown).

${ }^{152} \mathrm{Eu}$ solution $(30 \mathrm{ml}, 30 \mathrm{kBq})$ was added to a container, and its $\mathrm{pH}$ adjusted $(8.8 \pm 0.1)$. Following the $\mathrm{pH}$ adjustment, colloid suspension was added to the ${ }^{152} \mathrm{Eu}$. This gave a total volume of ${ }^{152} \mathrm{Eu} /$ colloid suspension of $300 \mathrm{ml}$. At different pre-equilibration times, aliquots of the ${ }^{152} \mathrm{Eu} /$ colloid suspension was removed $(10 \mathrm{ml}, \times 3)$ from the main mixture, and conditioned Dowex resin was added $(1.4 \mathrm{~g})$. The resulting mixture was allowed to shake gently for one hour. After shaking, the sample was centrifuged (15 min, $4000 \mathrm{rpm}$ ) and a $1.5 \mathrm{ml}$ aliquot of the supernatant was removed for analysis for $\mathrm{Eu}$ content. After measurement, the aliquot was returned to the sample tube, the resin resuspended, and the sample stored for subsequent analyses using the same method.

\section{Results and discussion}

\section{Bulk bentonite dissociation}

Powder X-ray diffraction was used to show that the EDTA had no effect on the bentonite (data not shown). Figure 1 shows the dissociation of Eu(III) from bentonite with EDTA contact time as a function of pre-equilibration time of the Eu with the bentonite prior to addition of EDTA. The data are plotted as the natural log of the percentage of Eu remaining bound to the bentonite. Eu(III) dissociation followed a similar pattern for all preequilibration times. A large part of the $\mathrm{Eu}(\mathrm{III})$ (30-50\%) dissociated almost instantaneously from the clay.

For the experiments with pre-equilibration times less than 115 days, the Eu distribution between bulk bentonite and EDTA was within error of that at equilibrium between 20 and 100 days. That is, the amount of $\mathrm{Eu}$ remaining bound to the bentonite reached the value that would have been observed if the EDTA had been present from the start of the experiment before the Eu was introduced to the clay: This point is shown as the horizontal black line in Fig. 1. Hence, for these systems, there was 


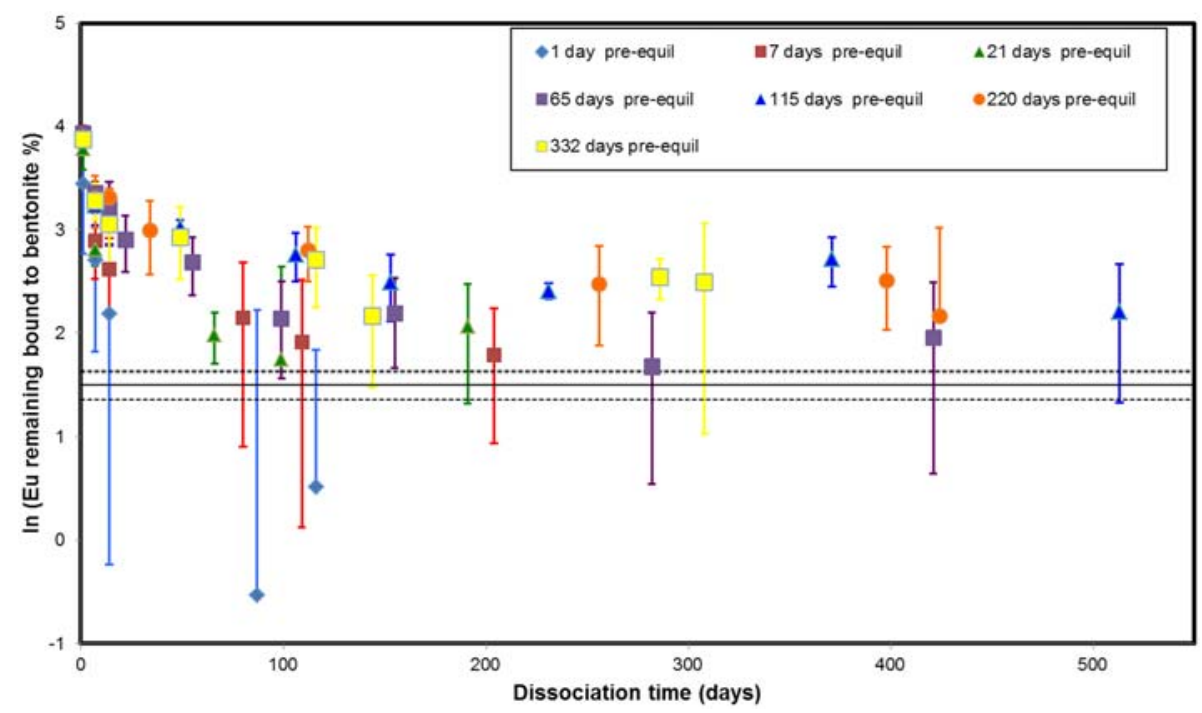

FIG. 1. Natural log of percentage of Eu bound to bentonite $v s$. EDTA contact time, as a function of pre-equilibration time $\left(\mathrm{pH}=7 \pm 0.1 ; \mathrm{I}=0.1 \mathrm{M} \mathrm{NaClO}_{4}\right)$. The full black horizontal line represents the equilibrium position, and the dashed lines represent the experimental uncertainty for the equilibrium position. Errors bars are $2 \sigma$ based on the standard deviation of triplicate measurements.

no indication of irreversibility. Experiments with pre-equilibration times above 115 days took longer to reach the equilibrium distribution, although all of them had at least one point within error of equilibrium. Therefore, there was no convincing evidence for irreversibility in these systems.

Linear portions of the plots in Fig. 1 indicate a single first-order dissociation rate constant, which may be determined from the gradient. Systems with pre-equilibration times greater than one day showed different behaviour to that of the one day system, where the dissociation was distinctly faster, with a smaller decrease in gradient as EDTA contact time progresses, compared to the other systems. The average dissociation rate constant for this system (taken from day one of EDTA contact until the system reaches apparent equilibrium) is approximately $10^{-6} \mathrm{~s}^{-1}$.

For the longer pre-equilibration times, each of the plots shows more than one gradient. There is faster dissociation at the start of the experiment, but after approximately seven days EDTA contact, there is a distinct reduction in reaction rate. First-order dissociation rate constants for the most slowly dissociating fraction were calculated by linear regression of the raw data for the portions of the plots beyond seven days of EDTA contact. The amount of $\mathrm{Eu}$ in the fraction was obtained from the intercept of the line of best fit with the $y$ axis.
The fitting results are shown in Table 1 . There are relatively small differences between the rates for the different systems. The average $\mathrm{Eu}(\mathrm{III})$ dissociation rate constant is $4.3 \times 10^{-8} \mathrm{~s}^{-1}$, with a range of $2.2 \times$ $10^{-8}-1.0 \times 10^{-7} \mathrm{~s}^{-1}$.

The Eu/EDTA/bentonite experiments are ternary systems (one metal ion, two sinks). Generally, the overall observed kinetics associated with metal exchange between one sink and another in such systems could be determined by the dissociation kinetics of the initial sink and the association kinetics of the receiving sink. For the receiving sink, the association rate would be expected to depend upon the binding site concentration. However, the association kinetics of $\mathrm{Eu}(\mathrm{III})$ and EDTA are very rapid, for example, Nash et al. (2012) found that the rate constant was $2.09 \times 10^{4} \mathrm{M}^{-1} \mathrm{~s}^{-1}$ in lactate systems. Therefore, at the EDTA concentrations used in this study, the rate of association of Eu(III) with EDTA is many orders of magnitude larger than the dissociation rate from the bentonite (Table 1). Therefore, the dissociation from the bentonite is the 'rate determining step', and the observed kinetics depend only on the rate of dissociation from the clay and are independent of the EDTA concentration.

The amount of $\mathrm{Eu}$ in the slowly dissociating fraction increases rapidly over the first few days of contact, but beyond 65 days, the amount seems to have reached an equilibrium, with around 19-25\% 


\section{REVERSIBILITY IN RADIONUCLIDE/BENTONITE TERNARY SYSTEMS}

TABLE 1 Dissociation rate constants, reaction half-time data and amounts for the most slowly dissociating fraction for $\mathrm{Eu}$ and bulk bentonite $\left(\mathrm{pH}=7 \pm 0.1 ; \mathrm{I}=0.1 \mathrm{M} \mathrm{NaClO}_{4}\right)$. Errors are $2 \sigma$ based on the error determined during regression of the data in Fig. 1 (due to the log vertical scale in Fig. 1, the errors on the amounts bound are asymmetric).

\begin{tabular}{lccc}
\hline $\begin{array}{l}\text { Pre-equilibrium time } \\
\text { (Days) }\end{array}$ & Dissociation rate constant $\left(\mathrm{s}^{-1}\right)$ & $\begin{array}{l}\text { Amount of Eu in slow dissociating } \\
\text { fraction with errors }(\%)\end{array}$ & $\tau$ (Days) \\
\hline 7 & $1.01 \times 10^{-7}\left( \pm 6.23 \times 10^{-8}\right)$ & $17.3(+3.1 ;-2.9)$ & 79 \\
21 & $4.19 \times 10^{-8}\left( \pm 8.51 \times 10^{-8}\right)$ & $11.9(+25.2 ;-4.8)$ & 192 \\
65 & $3.93 \times 10^{-8}\left( \pm 1.35 \times 10^{-8}\right)$ & $19.3(+5.4 ;-3.5)$ & 204 \\
115 & $2.17 \times 10^{-8}\left( \pm 1.70 \times 10^{-8}\right)$ & $20.5(+8.6 ;-6.0)$ & 370 \\
220 & $2.61 \times 10^{-8}\left( \pm 1.14 \times 10^{-8}\right)$ & $24.3(+6.7 ;-5.2)$ & 308 \\
332 & $2.56 \times 10^{-8}\left( \pm 2.87 \times 10^{-8}\right)$ & $20.7(+11.2 ;-7.3)$ & 314
\end{tabular}

slowly dissociating. Virtually all of the Eu in this system (>96\%) was bound to the bentonite before addition of EDTA; therefore, the other $75-81 \%$ of the $\mathrm{Eu}$ was bound to the bentonite, but did not get transferred to the slow fraction. The fact that this material was released instantaneously upon addition of EDTA shows that it was available for incorporation within a slowly dissociating fraction. Further, given the very low Eu concentration in the experiments, it seems unlikely that the slowly dissociating component has reached capacity. Therefore, in this system, there must be an equilibrium between the $\mathrm{Eu}$ in the slowly dissociating and instantaneously available fractions. There is an apparent decrease in the value of the rate constant obtained from regression as preequilibration time increases up to 115 days; however, there are large errors on the rate data.

When $\mathrm{Eu}$ was first added to the bentonite, it bound quickly, and within the first few hours of contact, $96 \%$ of the Eu was bound to the clay. Over the following month, there was a subsequent increase in the amount bound by $3.0 \%$ and an associated increase in the $R_{\mathrm{d}}$ by a factor of 4.1. It is clear that more than this $3.0 \%$ of the Eu changes its interaction with the bentonite, because there is different dissociation behaviour for the system with a short pre-equilibration time and those that were equilibrated for more than one day. Therefore, it seems that there is rapid uptake to some fraction that is bound, but may be (relatively) easily removed if a stronger sink becomes available. However, over time it seems that there is a transfer of some of the bound $\mathrm{Eu}$ to a fraction that dissociates more slowly. The small increase in $R_{\mathrm{d}}$ suggests that the transfer may also be associated with an increase in thermodynamic stability. However, some of the material (over half in most cases) also remains available for instantaneous dissociation, even after several months of contact time.

\section{Colloid size distributions}

The colloid and Eu/colloid mixture size distributions are shown in Fig. 2; nominally, the $<3 \mathrm{kDa}$ fraction will contain particles no larger than $\sim 1 \mathrm{~nm}$, and so this fraction can be considered as the 'true solution'.

The vast majority of the bentonite colloids were in nominal size fractions $>200 \mathrm{~nm}$. For the $3 \mathrm{k} \mathrm{Da}-$ $200 \mathrm{~nm}$ fraction, the ratio of $\mathrm{Al}: \mathrm{Mg}(0.62: 1$, with $\mathrm{Al}$ $=0.065 \mathrm{ppm}$ and $\mathrm{Mg}=0.105 \mathrm{ppm})$ was far from that of the bentonite theoretical value $(5.02: 1)$, and so this fraction does not seem to contain significant quantities of bentonite colloids. Also, the $\mathrm{Al}$ and $\mathrm{Mg}$ concentrations in this fraction were low compared to the others, and so a reliable bentonite colloid concentration could not be calculated. Given that the colloid stock solution had a concentration of $147 \mathrm{ppm}$, the material responsible for the $\mathrm{Al}$ and $\mathrm{Mg}$ in the $3 \mathrm{kDa}-200 \mathrm{~nm}$ fraction represents a very small percentage of the total. The ${ }^{152} \mathrm{Eu}$ (III) showed broadly similar behaviour to that of the colloid, and most of it was bound to the larger colloids $(>450 \mathrm{~nm} ; \approx 95 \%)$. Only $1.5 \%$ of the ${ }^{152} \mathrm{Eu}(\mathrm{III})$ remains in the true solution fraction $(<3 \mathrm{kDa})$. Therefore $>98.5 \%$ of the $\mathrm{Eu}$ is colloid associated. The $\mathrm{Eu}$ found in the colloidal fraction where no bentonite colloids were detected (10 kDa-200 nm) was probably due to Eu bound to low concentrations of smaller bentonite colloids that could not be detected or due to other trace colloidal material derived from the bentonite clay, such as quartz. These phases could generate colloids that could bind 


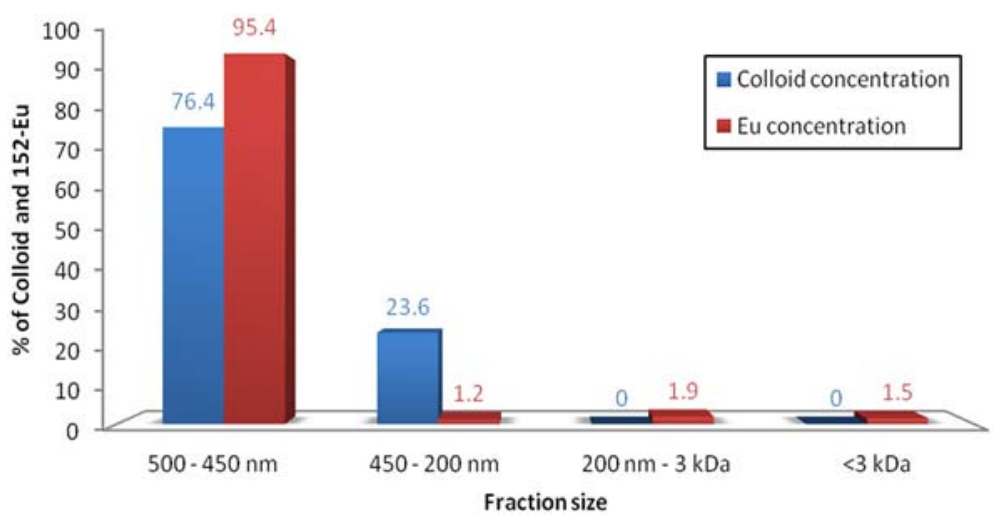

FIG. 2. Colloid and ${ }^{152} \mathrm{Eu}(\mathrm{III})$ concentrations in each size fraction.

$\mathrm{Eu}$, but they would not have the expected $\mathrm{Al}: \mathrm{Mg}$ ratio. Even a very low mass of colloids in the smaller fractions would be particularly reactive, because of their very high specific surface area. Despite this, the amount of Eu observed in the range $3 \mathrm{kDa}-200 \mathrm{~nm}$ is small, and so the ${ }^{152} \mathrm{Eu}(\mathrm{III})$ is largely associated with the bentonite colloids.

\section{Eu(III) colloid dissociation}

Sodium perchlorate was not added to the colloid experiments because this would have destabilized the colloids. Instead, the colloid solution was used without alteration. The dissociation of Eu(III) from the bentonite colloids as a function of Eu/colloid pre-equilibration time is shown in Fig. 3. In this system, when the Eu was added to the Dowex resin before the addition of colloids, the uptake was rapid, and $1.4 \pm 0.34 \%$ remained in solution; this represents the equilibrium position for the colloid experiments (horizontal line, Fig. 3). In this system, the colloid dissociation reaction is the rate-determining step. A description of the use of resins for the determination of dissociation rate constants is given in Monsallier et al. (2003). For all samples, a similar pattern is observed. A significant part of the $\mathrm{Eu}(\mathrm{III})$ (approximately $30-40 \%$ ) dissociated almost

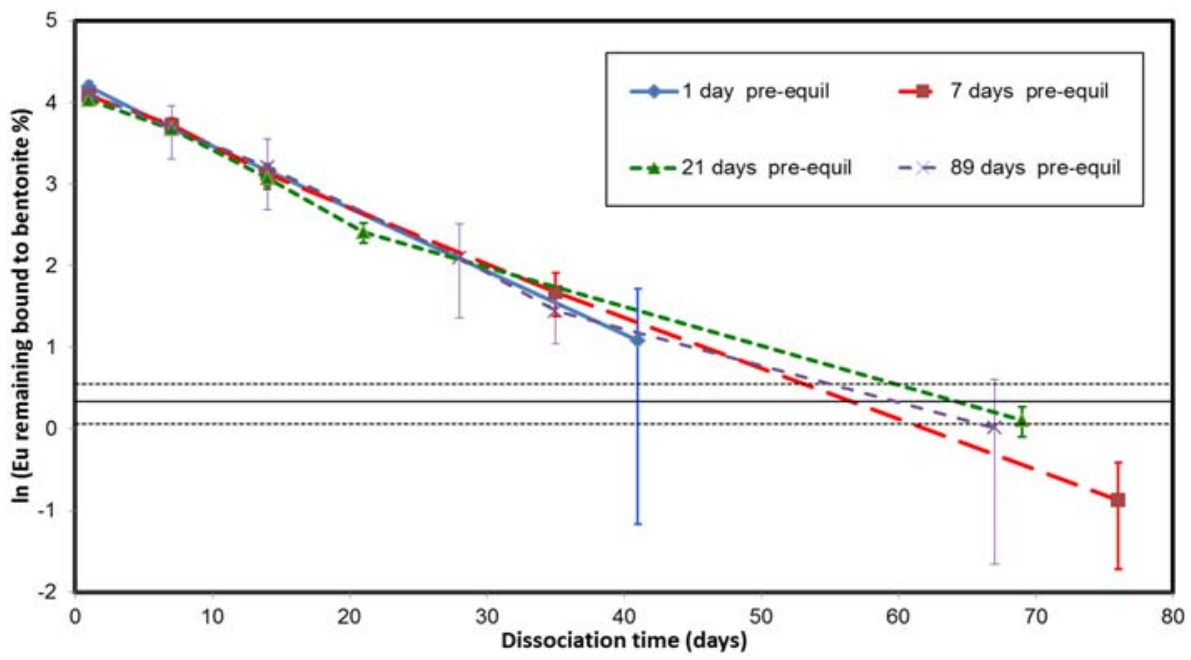

FIG. 3. Natural log plot of the colloid dissociation experiment: $\ln$ (percentage bound to bentonite) $v s$. Dowex resin contact times, as a function of pre-equilibration time $(\mathrm{pH}=8.8 \pm 0.1)$. The black horizontal line represents the equilibrium distribution, and the dashed lines represent the experimental uncertainty $(1.4 \pm 1.1 \%)$. 


\section{REVERSIBILITY IN RADIONUCLIDE/BENTONITE TERNARY SYSTEMS}

TABLE 2 Dissociation rate constants, reaction half-time data and amounts for the most slowly dissociating fraction $(\mathrm{pH}=8.8 \pm 0.1)$. Note, calculating an overall rate for all data in Fig. 3 gives an average first-order rate constant of $8.8 \times 10^{-7} \mathrm{~s}^{-1}$. Errors are $2 \sigma$ based on the error determined during regression of the data in Fig. 3 (due to the log vertical scale in Fig. 3, the errors on the amounts bound are asymmetric).

\begin{tabular}{lclc}
\hline Pre-equilibration system (Days) & Dissociation rate constant $\left(\mathrm{s}^{-1}\right)$ & Amount of Eu in fraction (\%) & $\tau$ (Days) \\
\hline 1 & $8.97 \times 10^{-7}\left( \pm 2.27 \times 10^{-8}\right)$ & $69.8(+3.1 ;-2.9)$ & 8.94 \\
7 & $7.66 \times 10^{-7}\left( \pm 5.63 \times 10^{-8}\right)$ & $60.8(+12.4 ;-10.3)$ & 10.5 \\
21 & $9.47 \times 10^{-7}\left( \pm 2.63 \times 10^{-7}\right)$ & $64.9(+22 ;-17)$ & 8.47 \\
89 & $9.04 \times 10^{-7}\left( \pm 2.55 \times 10^{-8}\right)$ & $69.2(+11 ;-9.5)$ & 8.87
\end{tabular}

instantaneously from the colloid. This was followed by slower dissociation of the remaining Eu(III) over time.

The dissociation observed for the colloids was very different to that of the bulk bentonite. More Eu is found in the slowly dissociating fraction, and there is no increase in the amount bound beyond one day pre-equilibration time. Further, beyond the initial rapid dissociation, only a single rate constant is observed throughout the duration of the experiment. First-order dissociation rate constants and the amounts in the most slowly dissociating component were calculated by regression, and the results are shown in Table 2.

The dissociation rate constants for the colloids are over an order of magnitude higher than for the bulk sample. The reasons for the differences are uncertain, but it could be due to the narrow size distribution of the bentonite colloids, making the system more homogenous than bulk experiments (Fig. 2). Beyond being more heterogeneous in terms of particle size, as a natural material, the bulk sample is also more chemically heterogeneous too. It seems likely that both of these factors contribute to the difference.

The binding of $\mathrm{Eu}(\mathrm{III})$ to bentonite in this experiment was similar to that reported in the literature. Missana et al. (2008) and Bouby et al. (2011) found that it would associate to the clay, but in the presence of a competitor, it would dissociate. Bouby et al. (2011) used humic acid as a competitor and reported that on addition of humic acid an instantaneous, partial dissociation of the radionuclide from the bentonite occurred. The same was observed in these experiments, since on initial addition of EDTA/resin at least $30 \%$ of the Eu dissociates immediately.

Wold (2010) estimated dissociation rate constants for some metal ions (see values above). These values were calculated from $K_{\mathrm{d}}$ values and association rates. We might expect some differences between the values reported here and those of Wold (2010) because Wold's calculation assumes that all of the bentonite-bound radionuclide represents a single fraction, whereas the kinetic data for the bulk and colloidal experiments (Figs 1 and 3) show at least 3 and 2 fractions, respectively. Wold's rate constant for $\mathrm{Am}$ (III) $\left(5.6 \times 10^{-7} \mathrm{~s}^{-1}\right)$ is an order of magnitude higher than that recorded here for the bulk $\left(4.3 \times 10^{-8} \mathrm{~s}^{-1}\right)$. The other trivalent rate constant from Wold (2010), Cm(III) (1.7 × $\left.10^{-6} \mathrm{~s}^{-1}\right)$, is also higher than the $\mathrm{Eu}$ bulk experiment rate here $\left(4.3 \times 10^{-8} \mathrm{~s}^{-1}\right)$. However, the colloid rate constants observed here fall within the range of trivalent rate constants reported by Wold (2010). Huber et al. (2011) have also provided dissociation rate constants for bentonite colloids from competition experiments using fracture-filling material. For Am(III), the values were in the range $1-2.5 \times 10^{-6} \mathrm{~s}^{-1}$, whilst for $\mathrm{Pu}(\mathrm{IV})$, the range was $3.9 \times 10^{-7}-2.4 \times 10^{-6} \mathrm{~s}^{-1}$. The bulk rate constants in this study are lower than the data from Huber et al. (2011) (Table1), but again the colloidal values are much closer (Table 2).

At present, the mechanism responsible for the slow dissociation is uncertain. For a lanthanide ion, such as Eu(III), we would not automatically expect dissociation from a surface complexation site to be so slow. Therefore, it seems that there must be some other mechanism responsible for the slow dissociation. In the case of some radionuclides and concentrations, it has been suggested that surface precipitation could be responsible, particularly for tetravalent ions (Bouby et al., 2011). However, it seems less likely that is the case for a trivalent radionuclide, such as Eu(III), especially given the low concentrations of $\mathrm{Eu}$ and the ambient $\mathrm{pH}$ studied here. Metal ions do diffuse into the interlayer spaces of clay structures, and it is possible that this is partly responsible for the slow dissociation: this might explain the difference 
between the bulk and colloidal $\mathrm{Eu}(\mathrm{III})$ rate constants, since the particles will be smaller for the colloid system, and so the extent to which a metal ion could get hidden inside the structure could be reduced, giving a faster dissociation rate. Clearly, additional work is required to deduce the mechanism of interaction more fully.

\section{Conclusions}

No evidence for 'irreversible' binding of Eu by bentonite has been observed. Although Eu(III) does bind to bulk bentonite and bentonite colloids and there was both a fast and slow dissociation, there was no sign that the radionuclides were bound permanently.

For bulk bentonite, beyond $\sim 100$ days preequilibration time, the amount of $\mathrm{Eu}$ in the most slowly dissociating fraction was effectively constant. However, the experiments suggest that there is a change in dissociation behaviour over preequilibration times up to 1 week, with a reduction in the slowest dissociation rate constant of approximately an order of magnitude. In addition to a significant fraction that dissociates instantaneously and some material with intermediate rates, there does seem to be a most slowly dissociating fraction that has a characteristic rate constant of approximately $4.3 \times 10^{-8} \mathrm{~s}^{-1}$.

There was a difference between the colloid and bulk dissociation. The first-order rate constant was significantly higher (i.e. faster reaction rate) for colloids, with an average dissociation rate constant of $8.8 \times 10^{-7} \mathrm{~s}^{-1}$, over an order of magnitude faster than for bulk bentonite. There was also only a single rate constant in the colloid system, and the amount slowly dissociating did not increase beyond a pre-equilibration time of one day. Previous work with humic substances has shown that in a system where some portion of colloid-associated radionuclides dissociate slowly, this will dominate transport (Bryan et al., 2007). Whether or not the rate constants measured here mean that colloidal transport is significant will depend upon transport residence times in the subsurface.

\section{Acknowledgements}

The research leading to these results has received funding from the European Atomic Energy Community's Seventh Framework Programme (FP7/ 2007-2011) under grant agreement number 295487, The BELBaR project. NB would also like to thank the
National Nuclear Laboratory for Strategic Research funding.

\section{References}

Bouby, M., Geckeis, H., Lutzenkirchen, J., Mihai, S. and Schafer, T. (2011) Interaction of bentonite colloids with $\mathrm{Cs}$, Eu, Th and $\mathrm{U}$ in presence of humic acid: A flow field-flow fractionation study. Geochimica et Cosmochimica Acta, 75, 3866-3880.

Bryan, N.D., Jones, D.L.M., Keepax, R.E., Farrelly, D.H., Abrahamsen, L.G., Warwick, P. and Evans, N. (2007) The role of humic non-exchangeable binding in the promotion of metal ion transport in the environment. Journal of Environmental Monitoring, 9, 329-347.

Comans, R.N.J. (1987) Adsorption, desorption and isotopic exchange of cadmium on illite: evidence for complete reversibility. Water Research, 21, 1573-1576.

Comans, R.N.J., Haller, M. and Depreter, P. (1991) Sorption of cesium on illite-nonequilibrium behavior and reversibility. Geochimica Cosmochimica Acta, 55, 433-440.

de Koning, A. and Comans, R.N.J. (2004) Reversibility of radiocaesium sorption on illite. Geochimica et Cosmochimica Acta, 68, 2815-2823.

Geckeis, H., Schäfer, T., Hauser, W., Rabung, T., Missana, T., Degueldre, C., Möri, A., Eikenberg, J., Fierz, T. and Alexander, W.R. (2004) Results of the colloid and radionuclide retention experiment (CRR) at the Grimsel test site (GTS), Switzerland - impact of reaction kinetics and speciation on radionuclide migration. Radiochimica Acta, 92, 765-774.

Huber, F., Kunze, P., Geckeis, H. and Schäfer, T. (2011) Sorption reversibility kinetics in the ternary system radionuclide-bentonite colloids/nanoparticles - granite fracture filling material. Applied Geochemistry, 26, 2226-2237.

Ivanov, P., Griffiths, T., Bryan, N.D., Bozhikov, G. and Dmitriev, S. (2012) The effect of humic acid on uranyl sorption onto bentonite at trace uranium levels. Journal of Environmental Monitoring, 14, 2968-2975.

Missana, T., Alonso, Ú., García-Gutiérrez, M. and Mingarro, M. (2008) Role of bentonite colloids on europium and plutonium migration in a granite fracture. Applied Geochemistry, 23, 1484-1497.

Monsallier, J.M., Schuessler, W., Buckau, G., Rabung, T., Kim, J.I., Jones, D., Keepax, R. and Bryan, N. (2003) Kinetic Investigation of Eu(III)-Humate Interactions by Ion Exchange Resins. Analytical Chemistry, 75, 3168-3174.

Möri, A., Alexander, W.R., Geckeis, H., Hauser, W., Schäfer, T., Eikenberg, J., Fierz, T., Degueldre, C. and Missana, T. (2003) The colloid and radionuclide retardation experiment at the Grimsel Test Site: 


\section{REVERSIBILITY IN RADIONUCLIDE/BENTONITE TERNARY SYSTEMS}

influence of bentonite colloids on radionuclide migration in a fractured rock. Colloids and Surfaces A: Physicochemical and Engineering Aspects, 217, 33-47.

Nash, K.L., Brigham, D., Shehee, T.C. and Martin, A. (2012) The kinetics of lanthanide complexation by EDTA and DTPA in lactate media. Dalton Transactions, 41, 14547-14556.

Pitois, A., Ivanov, P.I., Abrahamsen, L.G., Bryan, N.D., Taylor, R.J. and Sims, H.E. (2008) Magnesium hydroxide bulk and colloid-associated 152Eu in an alkaline environment: Colloid characterization and sorption properties in the presence and absence of carbonate. Journal of Environmental Monitoring, 10, 315-24.

Schäfer, T., Geckeis, H., Bouby, M. and Fanghänel, T. (2004) U, Th, Eu and colloid mobility in a granite fracture under near-natural flow conditions. Radiochimica Acta, 92, 731-737.

Wold, S. (2010) Sorption of prioritized elements on montmorillonite colloids and their potential to transport radionuclides. SKB Technical Report, TR10-20. SKB, Stockholm. 